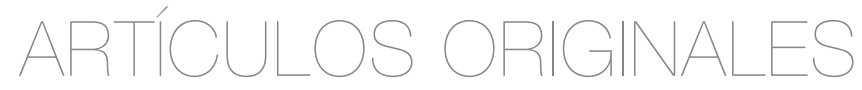

\title{
Gestão de projectos nas pequenas e médias empresas em Moçambique
}

ADALBERTO PAULINO FALSO ARMINDO*

* Doutorando em Gestao de Projectos e Gestao estrategica Empresarial. Instituto Superior de Ciência e Educação à Distância -ISCED-, Beira, Moçambique. E-mail: adarmindo@isced.ac.mz. ORCID: 0000-0001-8300-715X. Google Scholar: https://scholar.google.cl/ citations? user=rwUmStIAAAAJ\&hl=es. 
COMO CITAR ESTE ARTÍCULO

How to cite this article:

Falso, A. (2021). Gestão de projectos nas pequenas e médias empresas em Moçambique. Revista Perspectiva Empresarial, 8(2), 38-47.

Recibido: 11 de junio de 2021 Aceptado: 08 de octubre de 2021
RESUMO A relevância social e económica das pequenas e médias empresas para Moçambique e para outros quadrantes do mundo são notáveis. As investigações realizadas indicam que muitas empresas foram abertas e encerradas nos últimos 5 cincos anos, sendo fundamentais a fase inicial da atividade e os momentos de crise político-econômica. Objetivo. 0 presente artigo tem como foco a gestão empresarial e a gestão de projecto para garantir a sua continuidade no mercado. Metodologia. A pesquisa é do tipo exploratória. Abordagem usada foi mista (qualitativa e quantitativa) para determinar a amostra. E as técnicas de recolha de dados foram: a observação; a entrevista; análise documental; pesquisa bibliográfica e o questionário. Resultados. Este trabalho traz como resultados um novo modelo de administração nas pequenas e médias empresas moçambicanas. Os resultados obtidos contribuem com suporte teórico e prático para lideranças das pequenas e médias empresas moçambicanas e linhas de continuidade em estudos posteriores neste campo e em outras áreas. Conclusões. 0 trabalho conclui que as pequenas e médias empresas não implementação a gestão de projectos na sua administração, por isso, não sendo competitivas no mercado nacional e internacional.

PALAVRAS CHAVE gestão de projectos, pequenas e médias empresas, gestão empresarial.

\section{Gestión de proyectos en pequeñas y medianas empresas de Mozambique}

RESUMEN La relevancia social y económica de las pequeñas y medianas empresas para Mozambique y para otras partes del mundo es notable. Las investigaciones realizadas indican que muchas empresas se abrieron y cerraron en los últimos cinco años, siendo fundamental la fase inicial de la actividad y los momentos de crisis político-económica. Objetivo. Analizar la gestión empresarialy la gestión de proyectos para asegurar su continuidad en el mercado. Metodología. La investigación es exploratoria. El enfoque utilizado fue mixto (cualitativo y cuantitativo) para determinar la muestra. Y las técnicas de recolección de datos fueron: observación; entrevista; análisis de documentos; investigación bibliográfica y el cuestionario. Resultados. Este trabajo trae como resultado un nuevo modelo de administración en las pequeñas y medianas empresas mozambiqueñas. Los resultados obtenidos contribuyen al apoyo teórico y práctico para líderes de pequeñas y medianas empresas mozambiqueñas y líneas de continuidad en estudios posteriores en este campo y en otras áreas. Conclusiones. El trabajo concluye que las pequeñas y medianas empresas no implementan la gestión de proyectos en su administración, por lo que no son competitivas en el mercado nacional e internacional.

PALABRASCLAVE gestión de proyectos, pequeñas y medianas empresas, gestión empresarial. 


\section{Project management in small and medium-sized enterprises in Mozambique}

ABSTRACT Small and medium-sized enterprises have a remarkable social and economic relevance in Mozambique and other parts of the world. Research shows that many businesses have opened and closed in the last five years, with the initial phase of activity and times of political-economic crisis being critical. Objective. To analyze business management and project management in order to ensure their permanence in the market. Methodology. The study is exploratory and the method used to select the sample is mixed (qualitative and quantitative). Observation, interviews, document analysis, bibliographic research and questionnaires were the techniques used to collect data. Results. This work reveals a new management model in Mozambican small and medium-sized enterprises. The results obtained contribute to theoretical and practical support for leaders of Mozambican small and medium-sized enterprises, as well as lines of continuity in future studies in this and other fields. Conclusions. The study concludes that small and medium-sized businesses do not implement project management in their administration and, as a result, are not competitive in the national and international markets.

KEYWORDS Project management, small and medium-sized enterprises, business management. 


\section{Introdução}

A relevância social e económica das pequenas e médias empresas para Moçambique e para outros quadrantes do mundo são notáveis. As investigações realizadas indicam que muitas pequenas e médias empresas foram abertas nos últimos 5 cincos anos, mas também o índice da mortalidade das pequenas e médias empresas no igual período e até aos dias dehoje têm sido muito elevada, fundamental na fase do início da actividade e em momentos de crises.

Hoje em dia, o jogo das pequenas e médias empresas é a sua sobrevivência e fazendo face aos seus concorrentes no mercado, e para que a tal sobrevivência acontece elas devem pensar e terem uma visão de astúcia e modelos contemporâneos. Onde os modelos contemporâneos possam criar um clima organizacional favorável para aceitar ideias inovadoras para seus processos de produção para que aconteça a produtividade. Barbosa, de Oliveira e Lobosco (2011) acrescentam que a inovação é abordada como um impulso relevante e deu uma nova visão sobre as pequenas e médias empresas no desenvolvimento socioeconómico das regiões e passando a serem parceiras das estratégicas para as grandes empresas.

Além disso, esses autores, salientam que as estratégias de inovação são importantes para a transformação do destino das muitas empresas por criarem oportunidades para concorrerem e atenderem as exigências no mercado (Barbosa, de Oliveira e Lobosco, 2011).

Neste meio tempo, a habilidade na inovação é condicionada por um grupo de factores tais como força da concorrência e o impulso da mudança nas tecnologias de comunicação e informação. Para ser consolidada solicitam das pequenas e médias empresas habilidades internas organizacionais e as suas relações com as outras empresas no mercado. Acrescentando, Machado, Mazzali e Palmisano (2015), as acções inovadoras são relevantes tanto para o mundo de concorrência, tanto para o fomento dos próprios países.

Segundo Decreto 44/2011 de 21 de Setembro do Ministério de Indústria e Comércio de Moçambique, considera-se as pequenas e médias empresas segundo o critério de número de colaboradores e volume de negócio (tabela 1).

Tabela 1. Resumo de definição de pequenas e médias empresas em Moçambique

\begin{tabular}{lll}
\hline \multicolumn{1}{c}{ Classificação } & n.o de trabalhadores & Volume de negócio (MZN) \\
\hline Micro-empresa & $1-4$ & 〈1'200000.00 \\
\hline Pequena empresa & $5-49$ & 1'200000.00 $\leq 144^{\prime} 700000.00$ \\
\hline Média empresa & $50-100$ & 14 '700000.00 $\leq 29$ '970000.00 \\
\hline
\end{tabular}

Fonte: elaboração própria por ele autor.

Esteves e Nohara (2011), acrescentam que os critérios e conceitos usados para classificarem as pequenas e médias empresas são importantes para auxílio e reflexão. E que não existem critérios de classificação, universalmente aceite, podendo ser conceituado pelo volume de vendas e pelo número de colaboradores.

A nível internacional, as pequenas e médias empresas desempenham um papel importante no mosaico económico. Mas observa-se muita mortalidade delas no mercado nacionais, e possuem grandes desafios de diversas ordens em termos de acesso ao mercado internacional, no âmbito da globalização (Esteves e Nohara, 2011).

As pequenas e médias empresas desempenham também um papel extremamente importante na economia de Moçambique, minimizando a pobreza da população. 
Para Vieira e Martins (2018), na gestão empresarial as tarefas estratégicas devem se ajustarem a missão da empresa, conduzidas por uma política de valores com aptidão de planear, gerir e alocar da melhor maneira os seus recursos financeiro, humano e material, enquanto a administração e gestão são processos de planificação, organização, liderança e monitoramento de recursos organizacionais extremamente importantes para o sucesso das empresas.

Tello-Gamarra e Vershoore (2015), acrescentam que para uma empresa crescer ela deve ser competente ou ter competências. Quando falamos de competências organizacionais, ela deve ser vista sob dois pontos vista: uma de estratégia que versa sobre as habilidades da organização para fazer face aos desafios do mercado para a sua continuidade e a outra de gestão de recursos humanos, onde são indicadas as habilidades de cada colaborador no fomento de ideias criativas ou inovadoras para os produtos e serviços da empresa. Onde as boas políticas e práticas de gestão de recursos humanos devem ser posto em evidência para atrair candidatos aptos para as vagas e criarem condições para fomento de ideias inovadoras e com trabalho em equipa irão partilhar os seus conhecimentos de modo a inovarem nos seus processos produtivos de modo a gerarem novos produtos e serviços para a satisfação do cliente e a sua fidelização.

Da Silva, de Albuquerque e Freire (2017), salientam que a gestão de pessoas devem trazer resultados para a organização. 0 mais importante na gestão de pessoas além das vantagens de indicadores da avaliação, são as ligações entre a eficiência dos processos com o alcance dos propósitos organizacionais.

De acordo com Vieira e Martins (2018), a conceção de estratégia e a sua execução são elementos chaves para o sucesso de uma determinada empresas, sendo também um assunto de extrema relevância para os estudiosos, investigadores, pesquisadores no mosaico de gestão eadministração das empresas procuram dar as suas respostas. Na gestão estratégica, as deliberações e acções de astúcia empresariais em relação ao seu concorrente são indicadores fundamentais para uma visão futura.
Na gestão estratégica é fundamental pôr em evidência os três elementos básicos e os mais importantes para o futuro da corporação; assim, são chamados de factores estratégicos: forças, fraquezas, oportunidades e ameaças - SWOT-.

A gestão de projectos evoluiu bastante e trouxe uma visão do ponto de vista estratégico para garantir a continuidade das organizações. Nos dias de hoje, a gestão de projectos é praticamente usada em todos os departamentos das organizações (Kerzner, 2017).

Machado, Mazzali e Palmisano (2015), define a gestão de projectos como uma actividade estruturada com um tempo determinado, com finalidade de estabelecer um novo produto ou serviço para a satisfação das partes interessadas. E a gestão de projecto como sendo uma operação de estudo e inovação, ela necessita de uma equipa multifacetada e motivada.

Actualmente, a gestão de projectos é reconhecida como uma série de processos que pode ser usada em cada projecto, independentemente de sua duração ou complexibilidade, do valor do projecto ou da sua exposição ao risco. Pode-se sempreargumentar que gerir projectos de execução de planeamento estratégico não é diferente gerir qualquer outro tipo de projecto. Machado, Mazzali e Palmisano (2015), acrescentam que as organizações aplicam modelos organizacionais que geram resultados e com menores burocracias para coordenarem actividades diárias de forma eficiente.

Os gerentes de projectos precisam pensar estrategicamente, em vez de tácita ou operacionalmente, e talvez eles tenham que passar da liderança tradicional de gestão de projectos para uma liderança estratégica dependendo da complexidade do projecto.

0 modelo matricial facilita distribuição das tarefas ao projecto dentro das empresas, onde os recursos organizacionais serão usados de forma racional entre mais líderes. Aqui o gerente de projectos deve possuir perfil de líder e de bom negociador, para evitarem conflitos de interesses (Machado, Mazzali e Palmisano, 2015).

Rezende e Cordeiro (2016), abordam que o desenho e gestão de projeto são componentes 
fundamentais para mecanismo de inovação nas empresas, de modo a garantirem a sua continuidade no mercado através de estudo de operações e inovações nos processos produtivos das empresas. As características da gestão e desenho de projetos são elementos fundamentais na inovação de produtos e serviços das organizações, sendo o mais importantes: gestão de escopo; gestão de tempo; gestão de custos; gestão da qualidade; gestão de recursos humanos; gestão das comunicações; gestão de riscos; gestão de aquisições e gestão de integração (Magno, 2018).

Machado, Mazzali e Palmisano (2015), reforçam de forma resumida que a gestão de projetos se concretiza nas seguintes etapas: criação de escopo; definição do que será realizado (produtos e serviços). Onde o escopo contempla duas etapas: estrutura analítica de projecto e estrutura organizacional do projecto. Seguindo coloca-se a malha de tarefas de projectos, segundo um cronograma de actividades a serem executadas no projecto. E no fim, a etapa de monitoramento do projecto com objectivo de garantir se as tarefas programadas estão a decorrer segundo o planificado. Para cada modelo de gestão de projectos selecionado é importante tirar as liccoes apreendidas para acautelar projectos futuros.

O artigo acrescenta um suporte teórico sobre a gestão empresarial e de projectos para as pequenas e médias empresas moçambicanas e linhas de sequência em estudos futuros.

\section{Metodologia}

Moçambique fica situado no sudoeste da África, entre os paralelos 1027' e 2652' de latitude Sul e entre os meridianos de 3012' e 4051' longitude Este. Limitado ao Norte pela Tanzânia, ao Este pelo Oceano Índico, ao Oeste por Malawi, Zâmbia, Zimbabwe e Suazilândia e ao Sul pela República da África do Sul. Toda a faixa costeira Este é banhada pelo Oceano Índico. Com $799380 \mathrm{~km}^{2}$. Com uma população cerca de 30 '832244 habitantes, com a taxa de crescimento populacional de $2,5 \%$, a taxa bruta de natalidade de $37,6 \%$, a taxa de mortalidade infantil de $67,4 \%$, a taxa de crescimento do PIB de 2,28 \%.
Neste artigo, a observação foi possível obter dados sobre o ponto de situação sobre o ambiente interno e externo das pequenas e médias empresas. As entrevistas informais foram realizadas para as pequenas e médias empresas. Para este trabalho, foi usado os seguintes documentos livros, artigos de revistas indexadas publicadas sobre o objecto da pesquisa. 0 procedimento bibliográfico tomou como base o arrolamento das teorias variadas conceituadas na matéria de gestão/administração de empresas. Os questionários de inquérito, onde foram aplicados nas pequenas e médias empresas sobre os seus aspectos socioeconómico, gestão estratégica empresarial e gestão de projectos.

Para este artigo foi escolhida a amostra probabilística casual simples, trabalhando com 98 pequenas e médias empresas, onde foram representadas pelos seus respetivos gestores ou proprietários.

A colecta de dados foi aplicada nos anos 2020 a 2021, com duas fases distintas. Onde a primeira etapa foi durante o mês de agosto 2020 com tarefas de pedidos de autorizações para recolha de dados nas pequenas e medias empresas e a segunda etapa em janeiro de 2021 foi a questões de testes e execuções de técnicas de recolhas de dados.

\section{Resultados e discussão}

As pequenas e médias empresas desempenham um papel importante no mosaico económico, sendo um importante catalisador do crescimento e desenvolvimento social e económico em Moçambique. Por estarem inseridas na sua maioria em todos os sectores de actividades económicas e são grandes responsáveis pela contribuição fiscal, alimentam maior quantidade de empregos, consequentemente, maior promotora de renda.

A presente pesquisa teve como amostra 98 colaboradores das pequenas e médias empresas, sendo 96 do sexo masculino e 2 do sexo feminino, onde predomina uma população do tipo adulta (36-59 anos). 


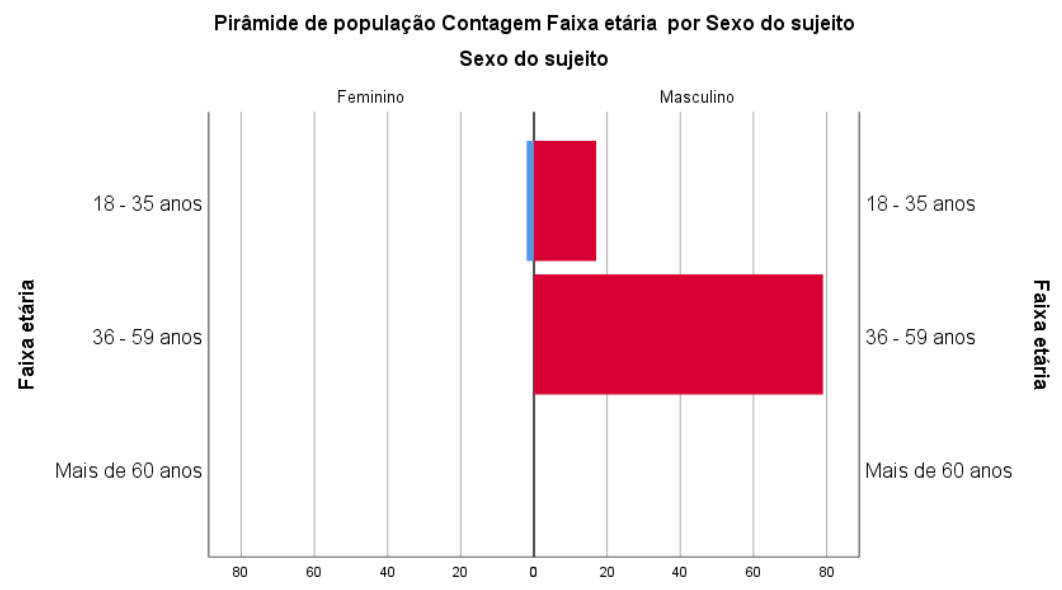

Figura 1. Pirâmide etária e sexual dos colaboradores das pequenas e médias empresas. Fonte: elaboração própria por ele autor.

Quanto às qualificações dos 98 colaboradores das pequenas e médias empresas, temos o seguinte: 17,3 \% formaram-se em gestão e comércio; $16,3 \%$ formaram-se em contabilidade e gestão; 9,2\% formaram-se em contabilidade e auditoria; 8,2 \% possuem formações nas áreas de direito, ensino geral e outras formações; 7,1 \% formaram-se em gestão de recursos humanos e por fim $2 \%$ possuem formação em bancaria e hoteleira. De modo geral os gestores possuem habilidades em gestão, onde temos 17,3 \% e 16,3 \% (gestão e comércio e contabilidade e gestão) (figura 2).

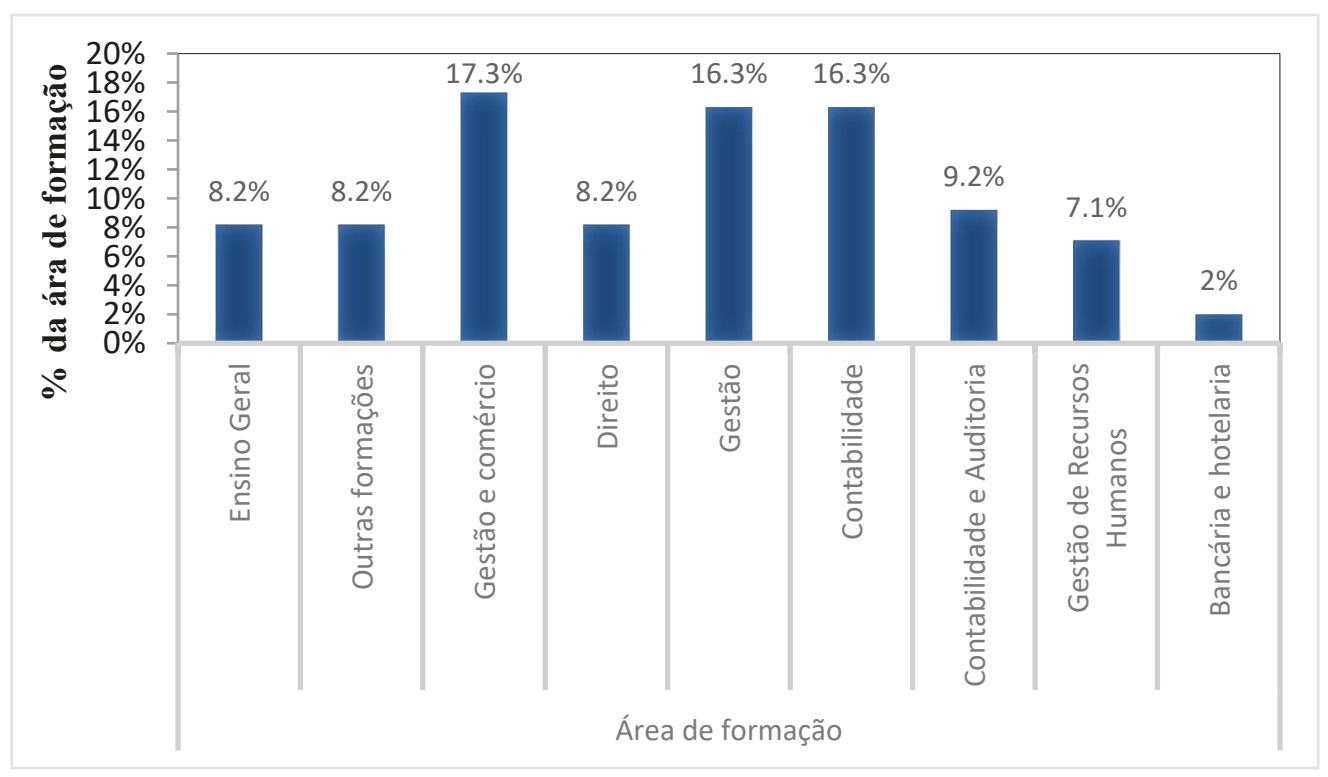

Figura 2. Áreas de formações dos gestores e proprietários das pequenas e médias empresas. Fonte: elaboração própria por ele autor. 
No que concerne às acções estratégicas das pequenas e médias empresas temos as seguintes realidades: 4,42 \% são representadas pelas acções estratégicas garantir a sobrevivência da organização; espaço virtual é apontada como via de comunicação entre empresa e cliente com ele 4,33\%; as novas tecnologias criam novas estratégias de negócio com ele 4,30 \%; a empresa investe em treinamento dos seus colaboradores com ele 3,62\%; o posicionamento estratégico baseia-se na variedade e inovação de produtos e serviços com ele 3,60\%; existe estratégia para enfrentar os desafios do mundo empresarial com ele 3,38 \%; existe uma gestão de relacionamento entre a organização e clientes com ele 3,35 \%; existe uma gestão de relacionamento entre a organização e clientes com ele $3,26 \%$; o processo de comunicação organizacional é eficiente e eficaz com ele 3,25\% e desenha-se projectos para resolução de problemas com ele 2,26\% (tabela 2).

Tabela 2. Acções estratégicas desenvolvidas pelas pequenas e médias empresas

\begin{tabular}{lccccc}
\hline & n.o & Mínimo & Máximo & Média & Erro Desvio \\
\hline Desenha-se projectos para resolução de problemas. & 97 & 1 & 5 & 2,26 & 1,285 \\
\hline Existe estratégia para enfrentar os desafios do mundo empresarial. & 97 & 1 & 5 & 3,26 & 0,992 \\
\hline $\begin{array}{l}\text { O posicionamento estratégico basea-se na variedade e inovação } \\
\text { de produtos e serviços. }\end{array}$ & 97 & 2 & 5 & 3,60 & 0,786 \\
\hline A empresa investe em treinamento dos seus colaboradores. & 98 & 1 & 5 & 3,62 & 0,831 \\
\hline Faz-se uma análise SWOT para a formulação de estratégia. & 97 & 2 & 5 & 3,38 & 0,847 \\
\hline As novas tecnologias criam novas estratégias de negócio. & 96 & 2 & 5 & 4,30 & 0,600 \\
\hline $\begin{array}{l}\text { Espaço virtual é apontada como via de comunicação entre empresa } \\
\text { e cliente. }\end{array}$ & 97 & 3 & 5 & 4,33 & 0,590 \\
\hline As acções estratégicas garantir a sobrevivência da organização. & 97 & 3 & 5 & 4,42 & 0,517 \\
\hline Existe uma gestão de relacionamento entre a organização e clientes. & 97 & 2 & 5 & 3,35 & 0,969 \\
\hline \begin{tabular}{l} 
O processo de comunicação organizacional é eficiente e eficaz. \\
\hline
\end{tabular} & 96 & 2 & 5 & 3,25 & 0,871 \\
\hline
\end{tabular}

Fonte: elaboração própria por ele autor.

Nas pequenas e médias empresas, onde na maioria das vezes são geridas pelos seus proprietários, as concepções de planos estratégicos, objectivos e processos de tomadas de decisões são segundo aos horizontes de próprios proprietários, onde se pode tirar ou serem identificadas os seguintes obstáculos atendendo e considerando a sua própria experiência, onde a concepção e implementação de acções estratégicas são derivadas a partir da execução de enormes tarefas e as suas vivência do dia-a-dia e acabando de dar menor importância a assuntos estratégicos.

Nesta senda, as pequenas e médias empresas ainda possuem lacunas para traçarem as suas próprias estratégias de modo a implementarem e avaliarem os resultados obtidos a partir da sua implementação. Por outro lado, a quando da formulação das referidas estratégias, não são tidas como um processo de racionalidade factuais, mas sim com as tomadas de decisões segundo as experiências e horizontes do proprietário. Porém, o processo de formulação de estratégias, não é acabado, mas sim continua licções aprendidas e retidas, para no futuro os proprietários tomarem conhecimentos e terem informações necessárias e relevantes sobre as habilidades e os pontos fracos, as incertezas e as oportunidades existentes no meio onde as suas organizações estão localizadas, além dos seus próprios mecanismos de produção. 
A avaliação do processo de formação de estratégias das pequenas e médias empresas, deve se tomar em conta a realidade das dimensões das empresas e diferentes abordagens da influência do tipo de liderança, dado a diferente relação de comunicação no processo de formulação de estratégias.

As pequenas e médias empresas quanto às acções estratégicas temos 4,42 \% são representadas pelas acções estratégicas que garantem a sobrevivência da organização e 2,26 \% que desenham projectos para resolução dos seus problemas organizacionais, mostrando que maior parte dos gestores ou proprietários não concebem nos seus planos de gestão o instrumento de desenho de projecto como metodologia para encontrar soluções robustas que podem se ajustar aos novos desafios do mercado.

Quanto a concepção teórica e prática sobre a gestão e o desenho do projecto em relação às áreas de formação dos gestores ou proprietário das pequenas e médias empresas, temos a seguinte realidade: os cursos de gestão e bancária e hoteleira com ele 3,9\%; cursos de contabilidade e gestão de recursos humanos com ele 3,8\%; curso de gestão e comércio com ele $3,7 \%$; cursos de contabilidade e auditoria e outras formações com ele 3,6\%; ensino geral com ele $3,4 \%$ e por último o curso de direito com ele 3,3\% (figura 3).

Segundo a realidade descrita acima, se concluiu que os colaboradores seniores das pequenas e médias empresas com as áreas de formações de gestão e bancária e hotelaria possuem mais conhecimentos sobre gestão e desenho de projectos com ele 3,9\%; os gestores ou proprietários das pequenas e médias empresas que não possuem noções sobre o desenho e gestão de projecto são os do ensino geral com ele $3,4 \%$ e os do curso de direito com ele $3,3 \%$.

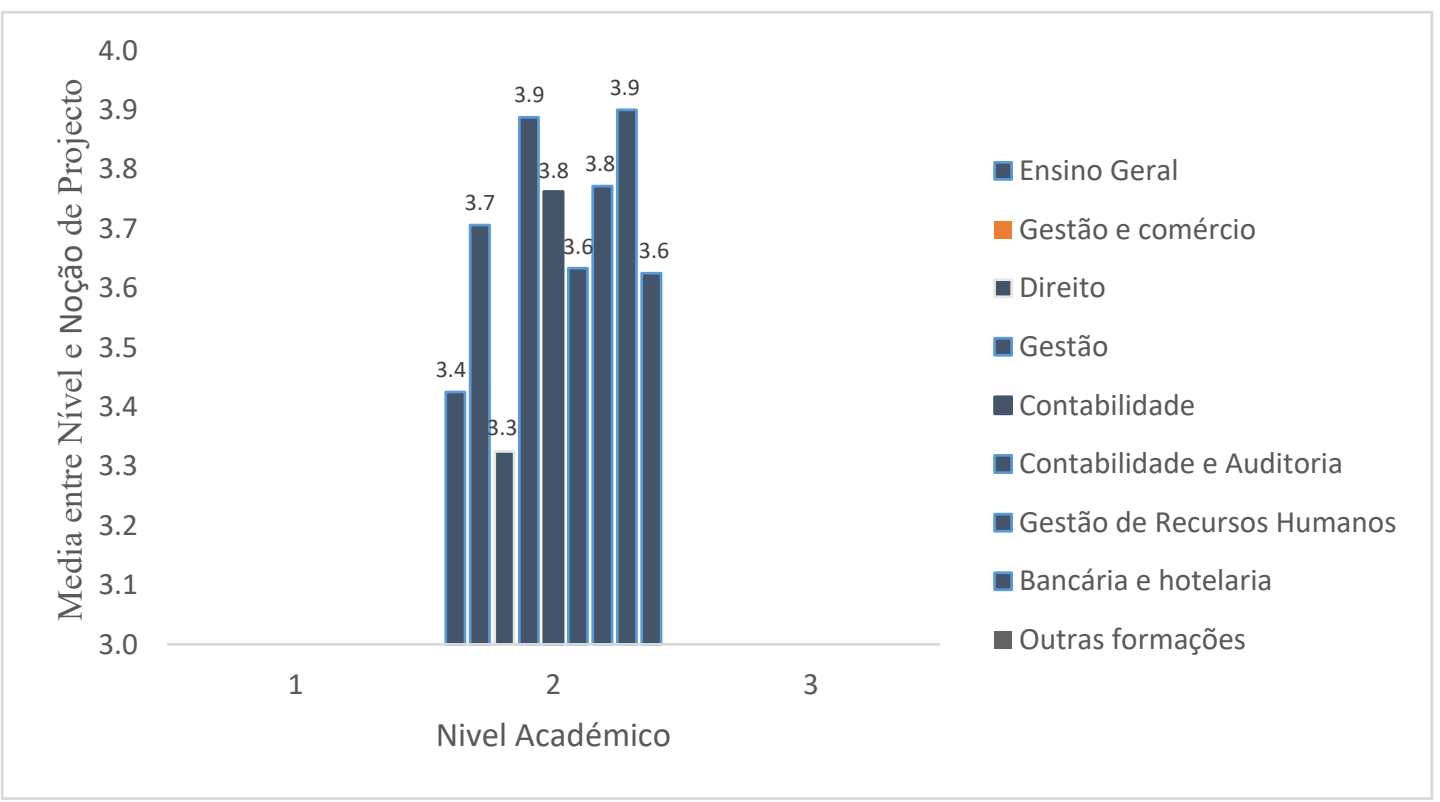

Figura 3. Noção de projectos segundo as áreas de formação dos gestores e proprietários das pequenas e médias empresas. Fonte: elaboração própria por ele autor. 
Essa gestão é apontada como um grande desafio para a maioria das empresas, visto que, a maioria dos problemas das pequenas e médias empresas explica-se pela falta de capacidade da gerência em resolver os desafios enfrentados no mundo de negócio. Onde o tipo de liderança é apontada como um elemento relevante para fomentar ideias inovadoras, criatividade virada do ponto de vista para a concepção dos objectivos e em certa medida centralizada na produção e a entrada em vigor de uma visão de invenções encorajadoras para as organizações retê-la e alimentá-la para o desenvolvimento da organização e para garantir a sua sobrevivência no mundo do negócio.

\section{Conclusão}

Terminado, foi notável queas pequenas emédias empresas desempenham um papel importante no mosaico económico, sendo um importante catalisador do crescimento e desenvolvimento social e económico de Moçambique. Outro dado extremamente importante alimenta maior quantidade de empregos, consequentemente, maior promotora de renda.

Os colaboradores seniores das pequenas e médias empresas com as áreas de formações de gestão e bancária e hotelaria possuem mais conhecimentos sobre gestão e desenho de projectos em detrimentos de outras áreas.

Às acções estratégicas das pequenas e médias empresas visam garantirem a sobrevivência das organizações.

Porém, a finalidade da pesquisa foi efectivada a partir da abordagem mista (quantitativa e qualitativa) na medida que as pequenas e médias empresas quanto às acções estratégicas na gestão não desenham projectos para resoluções robustas que podem se ajustar aos novos desafios do mercado.

\section{Referências}

Barbosa, M., de Oliveira, E. e Lobosco, A. (2011). Competências para inovar em pequenas e médias empresas tecnológicas. RAI: Revista de Administração e Inovação, 8(4), 206-212.

Da Silva, L.M., de Albuquerque, L.G e Freire, C.A. (2017). Capacidades organizacionais: um estudo na hotelaria do nordeste brasileiro. REGE-Revista de Gestão, 24, 170-180.

Esteves, G. e Nohara, J.J. (2011). Fatores críticos à estabilidade das alianças estratégicas das micros e pequenas empresas. RAI: Revista de Administração e Inovação, 8(3), 182-204.

Kerzner, H. (2017). Gestão de projectos, as melhores praticas. Porto Alegre, Brasil: Bookman.

Machado, C., Mazzali, L. e Palmisano, A. (2015). Gestão de projetos de inovação: 0 caso de uma empresa líder do setor de eletrodomésticos. RAI: Revista de Administração e Inovação, 12(3), 288-309.

Magno, C. (2018).As Balas de Prata no Gerenciamento de Projectos. Um Estudo em Projectos no Brasil. Lisboa, Portugal: Novas Edições Acadêmicas.

Rezende, R. eCordeiro, H. (2016). Estratégia, pessoas e operações como agentes influenciadores do desempenho do escritório de gerenciamento de projectos: uma análise por meio da modelagem de equações estruturais. Gestão \& Produção, 25(2), 410-429.

Tello-Gamarra, L. e Verschoore, J.R. (2015). 0 desenvolvimento das competências coletivas no terceiro setor. Suma de Negocios, 6, 194-203.

Vieira, P.e Martins, N.V.(2018). Fatores de impacto para sobrevivência de micro e pequenas (MPEs). Revista Livre de Sustentabilidade e Empreendedorismo, 3(5), 54-77. 